\title{
IREVIEW ARTICLE
}

\section{Current management and surgical advances in patients with hypertrophic obstructive cardiomyopathy}

Lucian Dorobantu, Razvan Ticulescu, Maria Greavu, Andrei Dermengiu, Maria Alexandrescu, Monica Trofin

Cardiomyopathy Center, Monza Hospital, Bucharest, Romania

\section{KEY WORDS}

hypertrophic cardiomyopathy, secondary chordae of the mitral valve, septal myectomy
Correspondence to: Lucian Dorobantu, MD, PhD, Cardiomyopathy Center, Monza Hospital, 27 Tony Bulandra Street, 021968 Bucharest, Romania, phone: +40312252500 , email: lucian.dorobantu@spitalulmonza.ro Received: August 29, 2019. Accepted: September 6, 2019. Published online: September 6, 2019. Kardiol Pol. 2019; 77 (9): 829-836 doi:10.33963/KP.14965 Copyright by the Author(s), 2019

\begin{abstract}
Hypertrophic cardiomyopathy $(\mathrm{HCM})$ is a genetic disease and the most frequent primary cardiomyopathy, affecting 1:500 of the general population. Integrated multimodality imaging, including transthoracic echocardiography, 2- and 3-dimensional transesophageal echocardiography, stress echocardiography, and cardiac magnetic resonance, has provided answers to questions on the management of HCM, leading to standardized protocols.

The late 1990s brought the news of a nonsurgical treatment of obstruction in HCM. It is now increasingly evident that septal ablation cannot address all the mechanisms of the left ventricular outflow tract (LVOT) gradient, especially mitral valve involvement. According to American and European guidelines, surgical septal myectomy is the current gold standard treatment. However, deep septal myectomy requires specific operator and institutional experience; therefore, it should not be performed in small community hospitals but only in centers of excellence for HCM treatment.

The so-called Ferrazzi technique involves cutting the fibrotic secondary chordae of the mitral valve (MV) and thus helps avoid a deep myectomy by moving the anterior mitral leaflet and the coaptation point of the MV posteriorly away from the septum. This technique, together with careful mobilization of the papillary muscles, helped us achieve excellent results since November 2015, with no mortality, resolution of the LVOT gradient, and MV preservation in all 72 patients.

Owing to recent advances in the surgical treatment of hypertrophic obstructive cardiomyopathy, addressing not only the septum but also the MV, the procedure of a deep myectomy has been simplified and mitral regurgitation adequately corrected.
\end{abstract}

Introduction Hypertrophic cardiomyopathy (HCM) is a genetic disease with a dominant autosomal transmission and an incomplete penetration. It is the most frequent primary cardiomyopathy, affecting 1:500 of the general population. 'The classic definition of HCM refers to a specific phenotype of increased wall thickness and left ventricular (LV) mass, whereas the sarcomeric HCM is known to be the major cause of unexplained LV hypertrophy. Nowadays, HCM is considered to represent an extremely heterogeneous pathology that determines a wide spectrum of phenotypic expression.
Pathophysiology and clinical presentation In the last years, there have been sustained efforts in the genetic field to elucidate the continuum between the genotype and phenotype of HCM as well as a treatment approach. To confirm a suspicion of HCM and a genotypic diagnosis, a genetic analysis is recommended (class of recommendation I, level of evidence B). ${ }^{2}$ In an overwhelming percentage of cases, the disease is caused by a single heterozygous mutation. In up to $75 \%$ of patients, the mutation appears in genes encoding myosin-binding protein $C$ and $\beta$-myosin heavy chain. ${ }^{3}$ The molecular and cellular mechanisms are not completely understood, 
but the disarray of the fibers, intercellular junction impairment, and replacement of cardiomyocytes with fibrotic tissue seem to be the main determinants of the pathogenesis. Thus, the clinical manifestation of HCM involves a wide spectrum of symptoms and severities, from asymptomatic or light effort dyspnea to syncope or sudden cardiac death, the latter being sometimes a single expression of the disease.

The new genetic data, suggesting such a great variation in disease expression, have provided a different perspective on the management of patients with HCM. The phenotypes of HCM (LV hypertrophy, LV outflow tract obstruction [LVOTO], fibrosis, myocardial ischemia, mitral regurgitation, and diastolic dysfunction) should be correlated with their clinical presentations heart failure, atrial fibrillation or stroke, angina, and sudden cardiac death.

In addition to the classic expression of $\mathrm{HCM}$ as LVOTO, a smaller proportion of patients will present atypical morphological patterns such as apical HCM, mid-cavity obstruction, or right ventricular hypertrophy. It is also clinically important to distinguish the minority of patients with significant LVOT obstruction but with a relatively mild septal hypertrophy (defined as a wall thickness exceeding $14 \mathrm{~mm}$ but lower than $18 \mathrm{~mm}$ ). This phenotype is very difficult to treat by invasive methods, and in 2015, Ferrazzi et $\mathrm{al}^{4}$ described a totally innovative approach to treatment.

Role of multimodality imaging in surgical decision making Integrated multimodality imaging, including transthoracic echocardiography
(TTE), 2- or 3-dimensional transesophageal echocardiography (TEE), stress echocardiography, and cardiac magnetic resonance (CMR), has helped identify multiple aspects of the various clinical presentations of HCM and has provided answers to management questions.

In 2015, the European Association of Cardiovascular Imaging expert consensus emphasized the important role of imaging in evaluating the risk of sudden cardiac death at 5 years, showing that half of the 8 criteria were based on a multi-imaging protocol. ${ }^{1}$ Integrated perioperative multimodality imaging techniques are crucial in surgical decision making. Comprehensive data such as the mechanism of LVOTO, the amount (extension, width, and depth) of the myocardium to be excised, and the anatomy and function of the mitral apparatus (and therefore the mechanism of mitral regurgitation) can all be assessed using standardized protocols for TTE, TEE, and CMR. In the recent years, preoperative CMR has played an increasing role in guiding the surgical planning.

Transthoracic echocardiography is recommended by consensus in all patients with HCM, and it should be done every 1 to 2 years in patients in stable condition. A TTE report in patients with HCM should contain standardized data about the LV diameter, thickness of the interventricular septum (IVS) and LV posterior wall in all segments, as well as the anterior-posterior diameter of the left atrium. Left ventricular volumes and ejection fraction should be calculated using the Simpson biplane method, and the LV mass, using the Devereux formula.
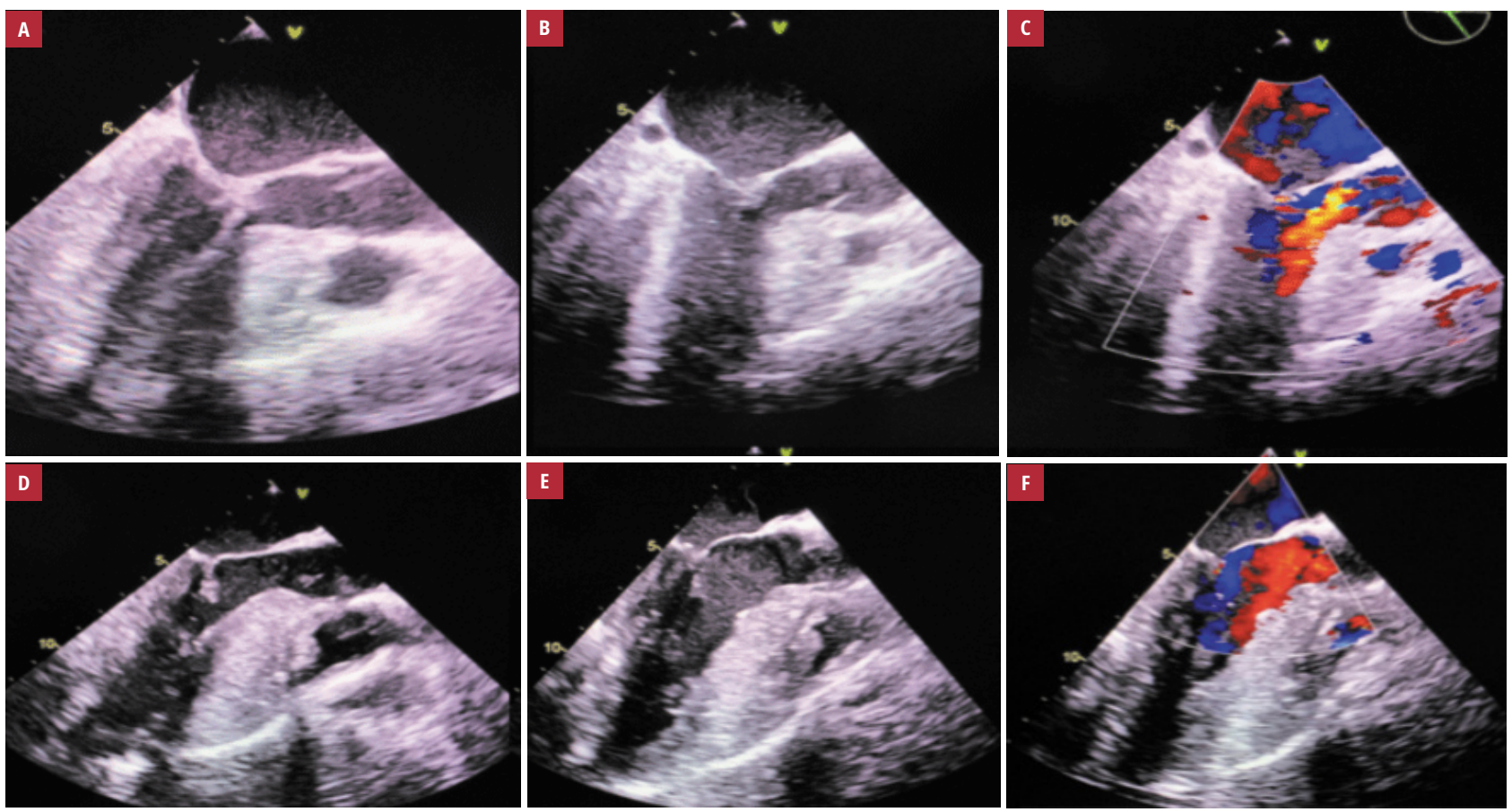

FIGURE 1 Results of transesophageal echocardiography: preoperative images showing left ventricular obstruction (A and $\mathbf{D}$ ); postoperative images in patient 1 (B and $\mathbf{C}$ ) and patient 2 (E and $\mathbf{F}$ ) showing resolution of the obstruction as a result of myectomy and cutting of fibrotic secondary chordae 
Peak systolic and peak early diastolic mitral annular velocities should be assessed from septal and lateral sites in order to estimate the filling pressures. The systolic anterior motion of the mitral valve (MV) is a hallmark of LVOTO, and its severity should be recorded. The anterior leaflet elongation and its mobility sometime determine mitral regurgitation, resulting in a posterior and lateral mitral regurgitation jet.

Color Doppler echocardiography is recommended for the semiquantitative evaluation of mitral regurgitation severity., ${ }^{5,6}$ If a central mitral regurgitation jet or multiple jets are observed, TEE should be performed to assess a particular mechanism of the mitral regurgitation. In HCM patients presenting with atrial fibrillation, the assessment of left atrial dysfunction (left atrial dimensions, volume, and remodeling) has shown to predict the clinical course of atrial fibrillation.

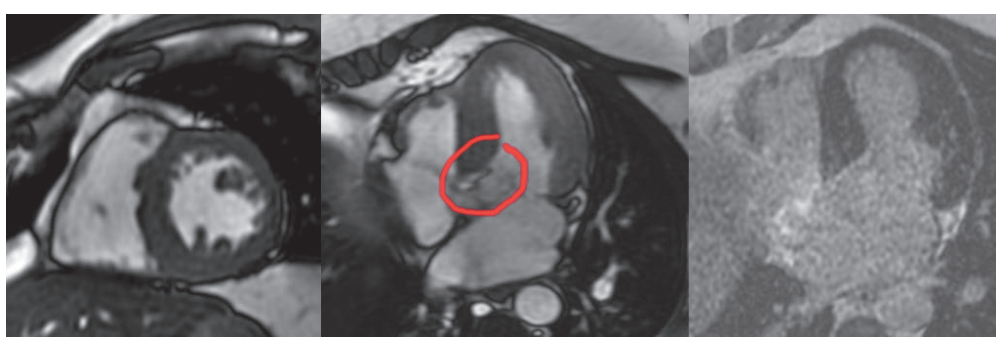

FIGURE 2 Cardiac magnetic resonance: mild septal hypertrophy seen in a short-axis view an obstructive gradient, proved by a turbulent flow (red circle) in the left ventricular outflow tract in a 3-chamber view, and no fibrosis seen on late gadolinium enhancement images

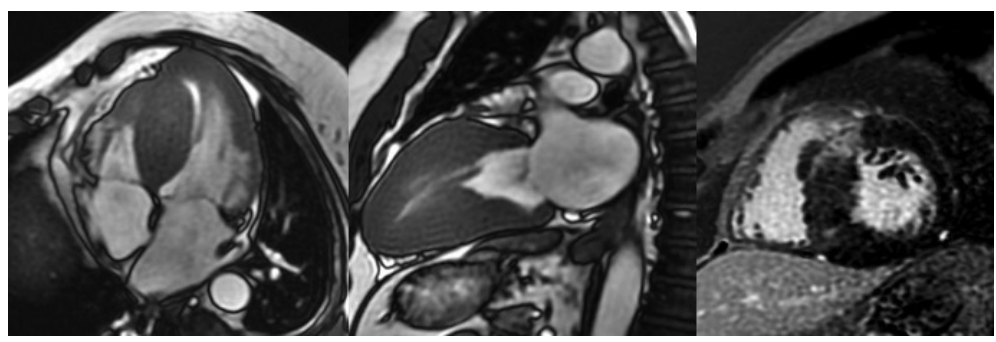

FIGURE 3 Cardiac magnetic resonance images showing obstructive hypertrophic cardiomyopathy: a 4-chamber and 2-chamber view showing extreme hypertrophy, predominantly of the septum; late gadolinium enhancement images showing myocardial fibrosis at the level of the anteroseptal wall

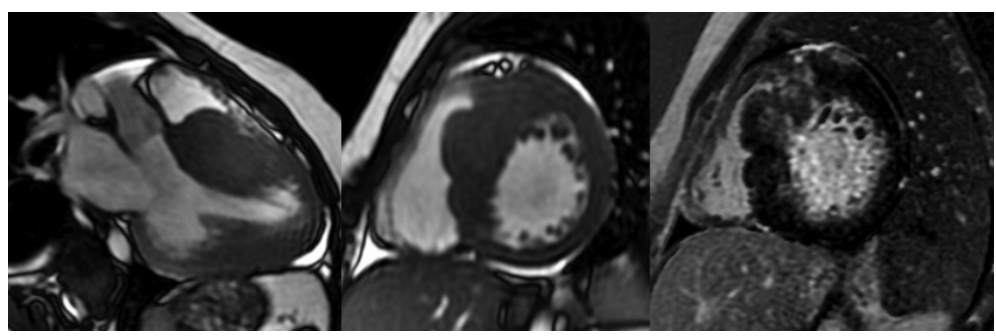

FIGURE 4 Cardiac magnetic resonance of a patient with evident obstructive hypertrophic cardiomyopathy: a 3-chamber and short-axis view showing significant fibrosis with diffuse distribution at the level of the entire interventricular septum
Obstruction is evaluated by measuring the LVOT gradient at rest or during stress conditions. Resting LVOT obstruction is defined as a peak gradient exceeding $30 \mathrm{~mm} \mathrm{Hg}$, and it is observed in up to one-third of patients with HCM. Another one-third of patients develop this gradient through Valsalva maneuvers or during stress. Stress echocardiography is used when a higher gradient is suspected, and it is assessed using an exercise protocol to decide on optimal management. Intraoperative TEE is performed to visualize the ventricular septum and MV apparatus. It is also used to accurately assess the maximal thickness septum for the myectomy, the apical extent of the septal excision, and MV abnormalities (such as annular calcifications, slack chordae, abnormal papillary muscle insertion, or fibrotic secondary chordae). Finally, it is the main modality used in the operating room to evaluate the early postprocedural outcome in order to confirm the absence LVOT obstruction, normal MV function, and the absence of iatrogenic complications (mainly an IVS defect) (FIGURE 1A-1F).

Cardiac magnetic resonance emerges as a unique method of tomographic reconstruction of the LV septum, due to its excellent spatial resolution. It offers a reliable quantification of LV ejection fraction in HCM and may aid the assessment of LV function in cases when the quality of an echocardiographic image is suboptimal or in the presence of atypical patterns. Moreover, current CMR techniques provide detailed anatomical and functional data for determining LVOTO, such as IVS geometry, flow direction, ${ }^{7}$ and the elements of the MV apparatus that may contribute to obstruction. Therefore, owing to its higher spatial resolution, CMR may improve the diagnosis of HCM by providing additional information when echocardiography is suboptimal or nondiagnostic (FIGURE2).

The late gadolinium enhancement (LGE) technique is based on the concept that tissue with an increased extracellular space will show a larger amount of a contrast agent, which is an extravascular substance. The current LGE algorithms provide such a high contrast-to-noise ratio that even small amounts of myocardial fibrosis can be visualized. Myocardial fibrosis is shown to be a strong independent predictor of cardiac outcome and evolution of the systolic function in patients with HCM. However, several studies have revealed that the adverse outcome is affected not by the pattern of distribution but rather by the total amount of LGE ${ }^{8,9}$ (FIGURES 3 and 4). T1 mapping is emerging as a promising method for the direct quantification of even very small or diffuse amounts of fibrosis, but research is ongoing on the use of T1 mapping in patients with HCM with no firm evidence yet.

Preoperative CMR has become increasingly used to guide surgical planning and to monitor invasive treatment procedures in HCM. It is 
already used in some centers as part of a standardized protocol to obtain a preoperative reconstruction of the septal anatomy in order to plan a strategy for septal myectomy. A limitation to the use of CMR, however, is the presence of a nonconditional cardiac implantable electronic device. Spirito et $\mathrm{al}^{10}$ described in detail the protocol of image acquisition and the concept of subsequent measurements at different levels of the ventricular wall (FIGURE 5).

In patients in whom CMR identifies myocardial crypts (narrow invaginations exceeding 30\% of the wall thickness of the adjacent myocardium with end-systolic collapse), the risk of iatrogenic septal defect at this level could be easily avoided. The prognostic relevance of CMR for this unique morphological variant of HCM is unknown, but the high-resolution capacity of CMR could help identify it also in family members of patients with HCM. ${ }^{10}$ The high spatial resolution of CMR also allows an assessment of anomalous muscle bundles, which are often located in the area of the septum where the wall thickness starts to decrease and connect the basal segment of the septum to its distal segment or to the free wall, potentially decreasing the mid-ventricular dimension. Understanding the anatomy of these aberrant muscle bundles could define a determinant of the obstruction itself, by displacing the papillary muscles in an anterior direction, towards the LV outflow tract.

Medical treatment The first-line treatment in symptomatic patients with LVOT obstruction is the use of classic nonvasodilating $\beta$-blockers, with the dosage titrated to the maximal tolerated dose. There was one study which showed that sotalol improved exercise tolerance in symptomatic patients with HCM. ${ }^{11}$ If the therapy is ineffective or not tolerated, disopyramide (when

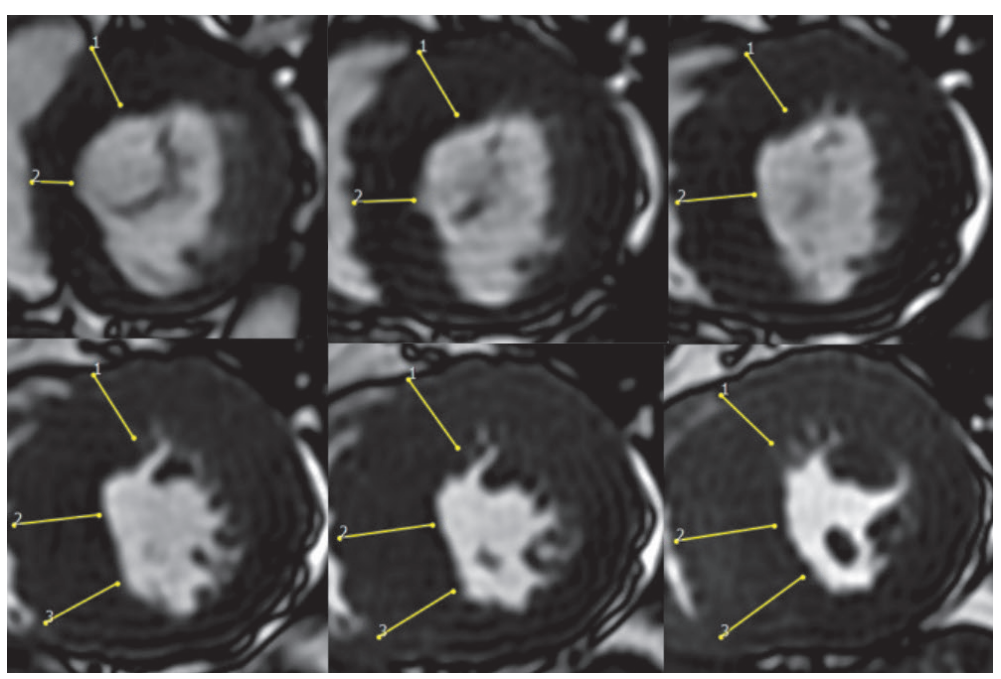

FIGURE 5 Preoperative cardiac magnetic resonance reconstruction of septal morphology, showing the measurements of the maximal septal thickness at different ventricular levels, using short-axis contiguous slices in a step-by-step process of reconstruction of thickness and length of the hypertrophied portion of the septum to be excised available) should be used, with special attention to patients with atrial fibrillation in whom disopyramide can enhance the ventricular rate. Another treatment option if $\beta$-blockers are not tolerated is nondihydropyridine calcium channel blockers. Verapamil and diltiazem should be carefully titrated, starting with low doses. However, despite improvement in symptoms, this therapy can result in pulmonary edema in patients with elevated systolic pulmonary artery pressure. Dihydropyridine calcium channel blockers are not recommended in the treatment of LVOTO. ${ }^{2}$ In patients with increased systolic pulmonary artery pressure, a diuretic therapy should be attempted. However, the use of diuretics is limited in HCM patients owing to the risk of reduction in the LV filling pressure and diastolic volume, which leads to an increase in the outflow gradient. In patients with atrial fibrillation, guideline recommendations should be followed, including the evaluation of the $\mathrm{CHA}_{2} \mathrm{DS}_{2}$-VASc score (congestive heart failure, hypertension, age $>75$ years, diabetes mellitus, history of stroke or thromboembolism, vascular disease, age 65 to 74 years, female sex) and then adjusting anticoagulant therapy accordingly. ${ }^{2}$

Dual-chamber pacing remains a controversial indication in the current guidelines (class of recommendation IIb, level of evidence B), ${ }^{2}$ the current literature being inconsistent.

Invasive treatment of the left ventricular gradient in hypertrophic obstructive cardiomyopathy There are 2 options for invasive therapy in patients with hypertrophic obstructive cardiomyopathy (HOCM): septal myectomy and catheter-based alcohol septal ablation. Both methods can be performed to relieve heart failure in patients with HCM with LV outflow obstruction and symptoms unresponsive to medical therapy. ${ }^{12-14}$ Both procedures require specific operator and institutional experience because $\mathrm{HCM}$ is seen relatively rarely in general cardiology practice..$^{10,13}$ Therefore, to achieve successful outcomes with a low complication rate, the procedures must be performed by highly trained cardiac surgeons and cardiologists.

For a long time, alcohol septal ablation was the treatment of choice for HOCM. This technique uses transcoronary ethanol administration via a percutaneous approach to reduce the outflow tract gradient by causing a localized septal infarction with septal thinning. However, the procedure has some limitations, most importantly the fact that it cannot repair MV abnormalities that contribute to outflow obstruction. It is also associated with numerous potential complications, including an increased risk of ventricular tachyarrhythmia due to septal scarring or a reduction in the septal thickness that cannot be predicted beforehand. Therefore, this procedure is indicated only in carefully 
selected patients with HCM, mainly those who are inoperable.

In the last years, the guidelines of American College and American Heart Association as well as those of the European Society of Cardiology have indicated septal myectomy as the gold standard treatment (ie, class I recommendation) for relief of LVOTO and severe heart failure symptoms in patients with HCM with marked gradients ( $\geq 50 \mathrm{~mm} \mathrm{Hg}$ ) at rest or with provocation and significant symptoms unresponsive to medications. ${ }^{10,15-17}$ More importantly, myectomy remains the only option in patients with outflow obstruction and associated abnormalities of the MV apparatus that require surgical correction. ${ }^{13,15,17,18}$

Due to the excellent results of the surgical treatment, Maron et a $1^{12}$ have recently suggested that despite the 2011 guidelines on HCM, myectomy should not be considered as the last therapeutic option for patients with severe symptoms, and, given the highly favorable benefit-to-risk ratio, providing a prolonged lifespan and a better quality of life, should be performed even in patients in the New York Heart Association functional class II.

Despite recommendations on surgical therapy, in Europe, the invasive treatment of LVOTO was mainly represented by alcohol septal ablation, including some countries, such as Germany and Switzerland, with previous important myectomy programs. ${ }^{19}$ During the last years, the surgical therapy of HCM in Europe has shown an increasing trend, mainly due to 2 reasons: the evidence of frequent failures of alcohol septal ablation as well as the abnormalities of the MV leaflets which contribute importantly to LVOTO in many (probably most) patients with HCM and can be resolved only by surgery. ${ }^{20-28}$

The most experienced European surgical centers are in Monza (Italy) ${ }^{20,21}$ and in Rotterdam (the Netherlands). ${ }^{20}$ They have developed major myectomy programs with results that are similar, in terms of operative mortality $(\leq 1 \%)$ and long-term clinical improvement and survival, to those of some of the most qualified centers in the United States, such as the Mayo Clinic and the Cleveland Clinic. ${ }^{12,13,15}$

In Bucharest, Romania, we started a myectomy program in 2015, and we have operated 72 patients so far, with no mortality and resolution of the LV gradient in all cases.

Evolution of surgical treatment: not only myectomy The first myectomy for obstructive HCM was performed in 1950 by Cleland et al. ${ }^{28}$ In the 1960s, Morrow et $\mathrm{al}^{25}$ proposed the transaortic resection of a small amount of muscle from the proximal ventricular septum, a technique generally described as classic myectomy.

For a short time, in the late 1970 s, replacement of the MV with prosthesis was proposed as an alternative to septal myectomy. ${ }^{26}$ Nowadays,
MV replacement is reserved for highly selected $\mathrm{HCM}$ patients with primary MV abnormalities not amenable to repair, ${ }^{28,29}$ due to significant risks associated with prosthetic valves, such as limited durability, infection, thromboembolism, and anticoagulation.

Over the past 30 years, myectomy has evolved from the classic Morrow operation to a more extended septal myectomy guided by preoperative cardiovascular magnetic resonance and intraoperative TEE. ${ }^{5,10,30,31}$ Extending the myectomy beyond the point of mitral-septal contact is important to obtain relief of the outflow gradient because failure to achieve this surgical target is routinely associated with a persistent gradient. ${ }^{32,33}$ Therefore, the "classic" myectomy requires relevant operator and institutional experience. ${ }^{2,32,34}$ As mentioned above, there are only a few myectomy centers in the world, mainly in the United States. In Europe, experienced centers are located in Italy, Belgium, the Netherlands, England, and Israel. 4,14,21 This is mainly because it is difficult to develop standard surgical approaches to the treatment of this disease, as it requires a deep septal myectomy and exposes patients to serious complications, such as postoperative ventricular septal defect or free wall rupture. Therefore, the current view is that myectomy should be discouraged in small community hospitals and should be performed only in"centers of excellence" for HCM treatment. ${ }^{35,15}$

On the other hand, there are no data on the learning curve for the operator. In 2017, 7 of the most experienced myectomy surgeons in the world published an expert opinion in which they stated that the number of septal myectomy surgeons is too low for the great number of patients who need this procedure. ${ }^{36}$

In recent years, various surgical approaches have been developed to correct MV abnormalities, including transaortic extension or plication of the anterior mitral leaflet. ${ }^{20,22,23}$ This was due to a wide range of $\mathrm{MV}$ abnormalities present in patients with obstructive HCM, such as elongated or calcified MV leaflets and fibrotic and/or retracted secondary chordae. All these abnormalities were demonstrated to be involved in the pathophysiology of the systolic anterior motion of the MV and outflow obstruction. ${ }^{12,20,31,37}$

In 2015, transaortic cutting of fibrotic secondary chordae, in combination with a shallow septal myectomy, was shown by Ferrazzi et $\mathrm{al}^{4}$ to move the MV apparatus to a more posterior position away from the septum, thereby augmenting the anterior MV surface, enlarging the outflow tract, preventing displacement of the MV into the LV outflow tract, and abolishing the outflow gradient. Such an approach was reported to improve outcomes in patients with obstructive HCM and severe heart failure. ${ }^{4}$ When we started a myectomy program in our center, we decided to use this technique in 


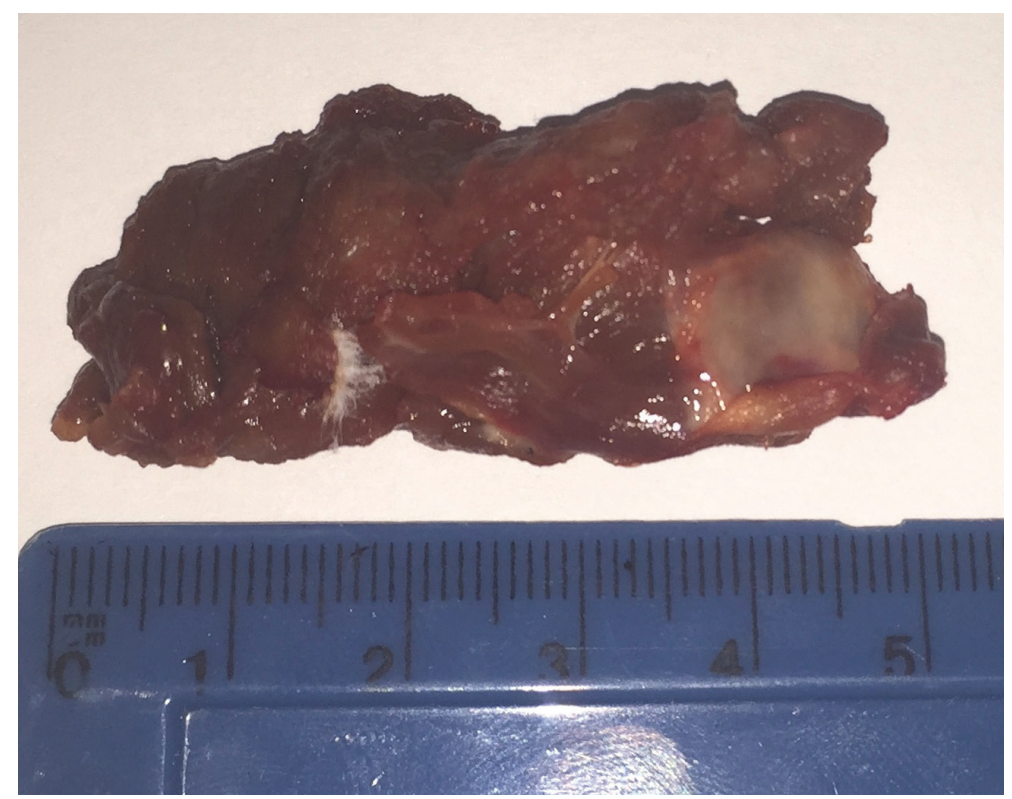

FIGURE 6 One-piece myectomy

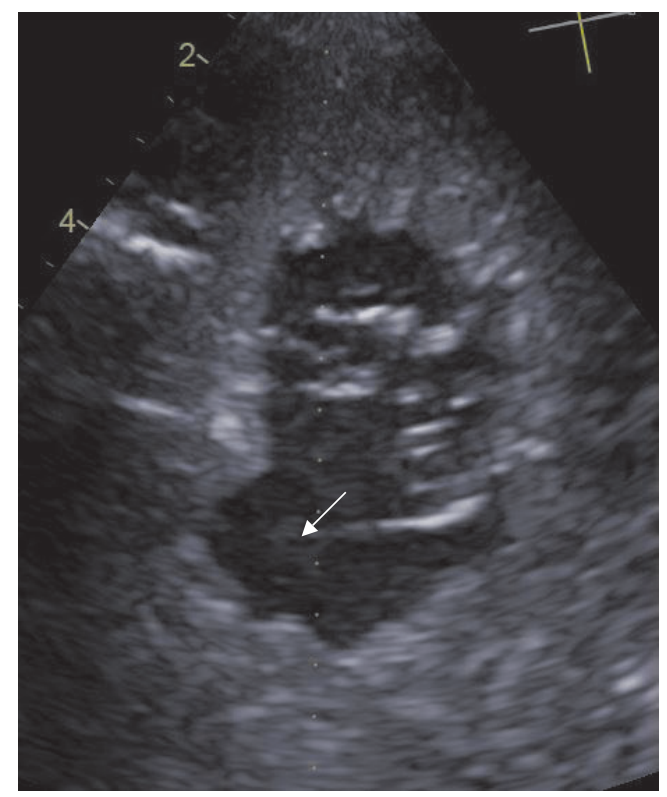

FIGURE 7 Result of postoperative transesophageal echocardiography after myectomy (arrow)

surgeon. This is another reason why we started the myectomy program in Romania using the technique by Ferrazzi et al. ${ }^{4}$ In all 72 patients operated at our institution, we used a "one piece" myectomy tailored at exactly one-third of the initial septal thickness, with particular attention to pass the equator of the LV whenever possible. The precise dimensions of the segment undergoing myectomy was selected based ator is essential for an inexperienced myectomy

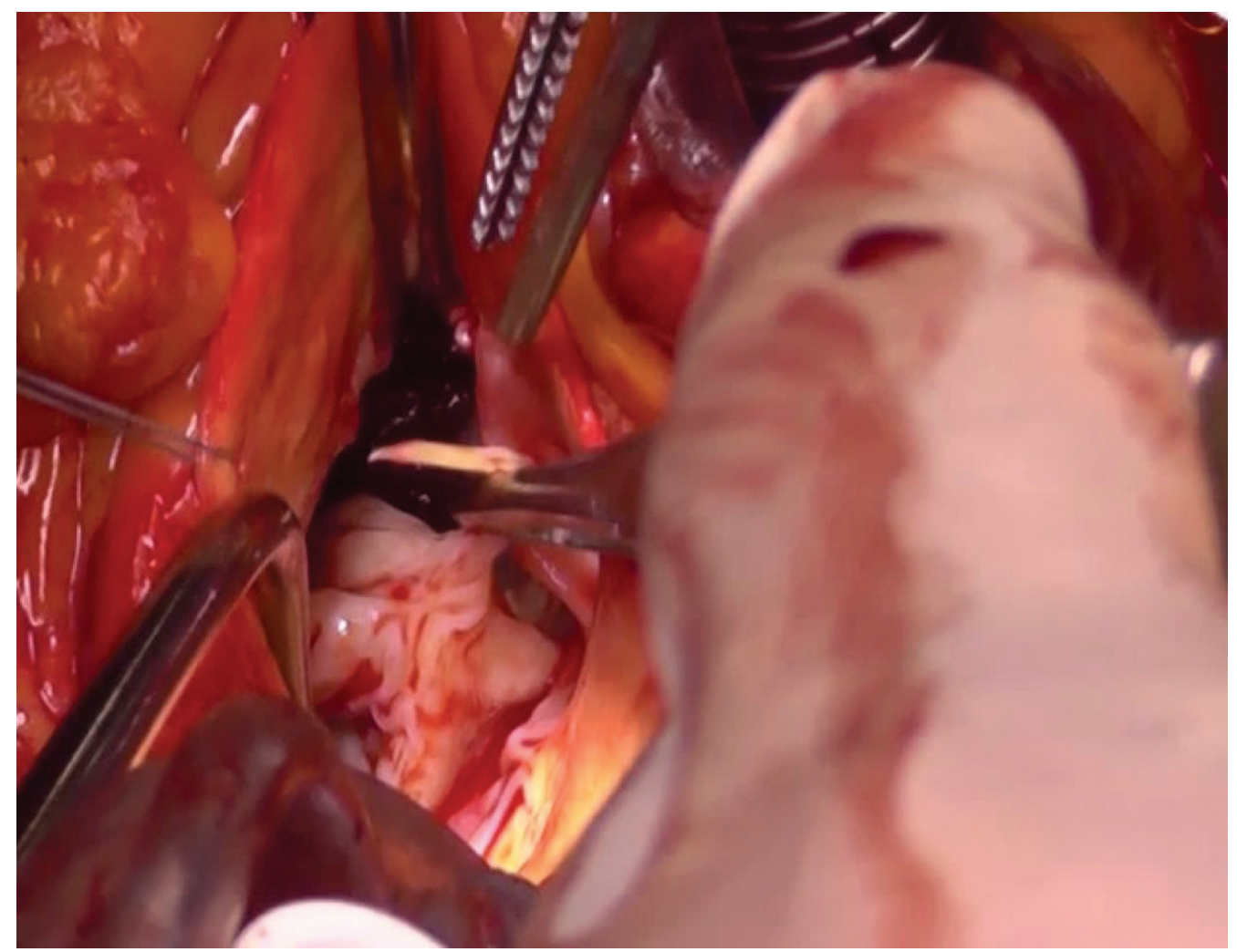

FIGURE 8 Intraoperative image showing a resected fibrotic secondary chordae 
on CMR, TEE, and TTE measurements. A careful mobilization of the papillary muscles and cutting of all fibrotic secondary chordae (FIGURE 8) allowed us to abolish the LV outflow gradient and preserve the MV in all patients, with no mortality and major complications.

Our experience and that of Dr. Ferrazzi with cutting secondary chordae in combination with a shallow septal myectomy show that this novel approach to surgical treatment of HOCM, which reduces the technical challenges of this procedure as well as the need for deep septal resection and / or MV replacement, could improve the access of patients with HOCM to septal myectomy.

Final remarks This review is an update on the management of patients with HOCM, with particular focus on recent advances in surgical treatment. As we acknowledge the complexity of data regarding the pathophysiology and phenotypic expression of this entity, we were unable to mention all the aspects of HCM management. New genetic data shed light on the heterogeneity of the disease variants even in the same family. The improvement in the understanding of its pathophysiology made it possible to correlate the phenotypes with their clinical presentations, which led to the development of standardized protocols including multimodality imaging techniques.

It is increasingly evident today that septal ablation is not able to address all the mechanisms of the LVOT gradient, especially MV involvement. Therefore, septal myectomy is the method of choice, with very good and reproducible results. As a result of recent advances in the complex surgical treatment of HOCM, addressing not only the septum but also the MV, the procedure of deep myectomy could be simplified and the mitral regurgitation properly corrected.

\section{ARTICLE INFORMATION}

\section{CONFLICT OF INTEREST None declared}

OPEN ACCESS This is an Open Access article distributed under the terms of the Creative Commons Attribution-NonCommercial-NoDerivatives 4.0 International License (CC BY-NC-ND 4.0), allowing third parties to download articles and share them with others, provided the original work is properly cited, not changed in any way, distributed under the same license, and used for noncommercial purposes only. For commercial use, please contact the journal office at kardiologiapolska@ptkardio.pl.

HOW TO CITE Dorobantu L, Ticulescu R, Greavu M, et al. Current management and surgical advances in patients with obstructive hypertrophic cardiomyopathy. Kardiol Pol. 2019; 77: 829-836. doi:10.33963/KP.14965

\section{REFERENCES}

1 Cardim N, Pettersen J, Popescu BA, et al. Role of multimodality imaging in the management of patients with hypertrophic cardiomyopathy - an expert consensus of the European Association of Cardiovascular Imaging endorsed by the Saudi Heart Association. Eur Heart J Cardiovasc Imaging. 2015; 16: 280 .

2 Elliott PM, Anastasakis A, Borger MA, et al. 2014 ESC guidelines on diagnosis and management of hypertrophic cardiomyopathy: the Task Force for the Diagnosis and Management of Hypertrophic Cardiomyopathy of the European Society of Cardiology (ESC). Eur Heart J. 2014; 35: 2733-2779.

3 Popa-Fotea NM, Micheu MM, Bataila V, et al. Exploring the continuum of hypertrophic cardiomyopathy's-from DNA to clinical expression. Medicina (Kaunas). 2019; 55: 299.

4 Ferrazzi P, Spirito P, Iacovoni A, et al. Transaortic chordal cutting: mitral valve repair for obstructive hypertrophic cardiomyopathy with mild septal hypertrophy. J Am Coll Cardiol. 2015; 66: 1687-1699.
5 Roşca M, Călin A, Beladan CC, et al. Right ventricular remodeling, its correlates ant its clinical impact in hypertrophic cardiomyopathy. J Am Soc Echocardiogr. 2015; 28: 1329-1338.

6 Roşca M, Popescu BA, Beladan CC, et al. Left atrial dysfunction as a correlate of heart failure symptoms in hypertrophic cardiomyopathy, J Am Soc Echocardiogr. 2010; 23: 1090-1098.

7 Śpiewak M, Kubik A, Kłopotowski M, et al. Four-dimensional flow magnetic resonance imaging in hypertrophic obstructive cardiomyopathy. Kardiol Pol. 2017; 75: 813.

8 Chan RH, Maron BJ, Olivotto I, et al. Prognostic value of quantitative contrast-enhanced cardiovascular magnetic resonance for the evaluation of sudden death risk in patients with hypertrophic cardiomyopathy. Circulation. 2014; 130: 484-495.

9 Ismail TF, Jabbour A, Gulati A, et al. Role of late gadolinium enhancement cardiovascular magnetic resonance in the risk stratification of hypertrophic cardiomyopathy. Heart. 2014; 100: 1851-1858.

10 Spirito P, Binaco I, Poggio D, et al. Role of preoperative cardiovascular magnetic resonance in planning ventricular septum myectomy in patients with obstructive hypertrophic cardiomyopathy. Am J Cardiol. 2019; 123: 1517-1526.

11 Tendera M, Wycisk A, Schneeweiss A, et al. Effect of sotalol on arrhythmias and exercise tolerance in patients with hypertrophic cardiomyopathy. Cardiology. 1993; 82: 335-342.

12 Maron MS, Spirito P, Maron BJ. Case for earlier surgical myectomy in patients with obstructive hypertrophic cardiomyopathy. Circulation. 2018; 138: 2076-2078.

13 Maron BJ, Rowin EJ, Casey SA, Maron MS. How hypertrophic cardiomyopathy became a contemporary treatable genetic disease with low mortality: shaped by 50 years of clinical research and practice. JAMA Cardiol. 2016; 1: 98-105.

14 Vriesendrop PA, Schinkel AF, Soliman OI, Kofflard MJ, de Jong PL, van Herwerden $L A$, et al. A contemporary European experience with surgical septal myectomy and anterior mitral vave extension in obstructive cardiomyopathy. Eur Heart J. 2012; 33: 2080-2087.

15 Kim LK, Swaminathan RV, Looser $P$, et al. Hospital volume outcomes after septal myectomy and alcohol septal ablation for treatment of obstructive hypertrophic cardiomyopathy: US Nationwide Inpatient Database, 2003-2011. JAMA Cardiol. 2016; 1: 324-332.

16 Desai MY, Smedira NG, Bhonsale A, et al. Symptom assessment and exercise impairment in surgical decision making in hypertrophic obstructive cardiomyopathy: relationship to outcomes. J Thorac Cardiovasc Surg. 2015; 150: 928-935. 17 Desai MY, Bhonsale A, Smedira NG, et al. Predictors of long-term outcomes in symptomatic hypertrophic obstructive cardiomyopathy patients undergoing surgical relief of left ventricular outflow tract obstruction. Circulation. 2013; 128: 209-216. 18 Woźnica A, Tyczyński P, Brzozowski P, et al. Hypertrophic obstructive cardiomyopathy with anomalous left circumflex coronary artery. Kardiol Pol. 2018; 76: 1118 19 Vriesendorp PA, Schinkel AF, Soliman OI, et al. Long-term benefit of myectomy and anterior mitral leaflet extension in obstructive hypertrophic cardiomyopathy. Am J Cardiol. 2015; 115: 670-675.

20 Iacovoni A, Spirito P, Simon C, et al. A contemporary European experience with surgical septal myectomy in hypertrophic cardiomyopathy. Eur Heart J. 2012; 33: 2080-2087.

21 Smedira NG, Lytle BW, Lever HM, et al. Current effectiveness and risks of isolated septal myectomy for hypertrophic obstructive cardiomyopathy. Ann Thorac Surg. 2008; 85: 127-133.

22 Balaram SK, Ross RE, Sherrid MV, et al. Role of mitral valve plication in the management of hypertrophic cardiomyopathy. Ann Thorac Surg. 2012; 94: 1990-1997.

23 Sherrid MV, Balaram S, MD, Kim B, et al. The mitral valve in obstructive hypertrophic cardiomyopathy: a test in context. J Am Coll Cardiol. 2016; 67: 1846-1858.

24 Goodwin JF, Hollman A, Cleland WP, Teare D. Obstructive cardiomyopathy simulating aortic stenosis. Br Heart J. 1960; 22: 403-414.

25 Morrow AG, Fogarty TJ, Hannah H 3rd, Braunwald E. Operative treatment in idiopathic hypertrophic subacute stenosis. Techniques and the results of preoperative and postoperative clinical and hemodynamic assessments. Circulation. 1968; 37: 589-596.

26 Cooley DA, Wukasch DC, Leachman RD. Mitral valve replacement for idiopathic hypertrophic subaortic stenosis. Results in 27 patients. J Cardiovasc Surg. 1976; 17: 380-387.

27 Maron B], Nishimura RA. Surgical septal myectomy versus alcohol septal ablation. Assessing the status of the controversy in 2014. Circulation. 2014; 130: 1167-1124.

28 Dearani JA, Ommen SR, Gersh B], et al. Surgery insight: septal myectomy for obstructive hypertrophic cardiomyopathy - the Mayo Clinic experience. Nat Clin Pract Cardiovasc Med. 2007; 4: 503-512.

29 Maron MS, Olivotto I, Harrigan C, et al. Mitral valve abnormalities identified by cardiovascular magnetic resonance represents a primary phenotypic expression of hypertrophic cardiomyopathy. Circulation. 2011; 124: 40-47.

30 Yang DH, Kang JW, Kim N, et al. Myocardial 3-dimensional printing for septal myectomy guidance in a patient with obstructive hypertrophic cardiomyopathy. Circulation. 2015; 132: 300-301.

31 Maron BJ, Yacoub M, Dearani JA. Controversies in cardiovascular medicine. Benefits of surgery in obstructive hypertrophic cardiomyopathy: bring septal myectomy back for European patients. Eur Heart J. 2011; 32: 1055-1058.

32 Ong KC, Geske JB, Hebl VB, et al. Pulmonary hypertension is associated with worse survival in hypertrophic cardiomyopathy. Eur Heart J Cardiovasc Imaging. 2016; 17: 604-610. 
33 33Spirito P, Ferrazzi P. Pulmonary hypertension in hypertrophic cardiomyopathy: a forgotten marker in the identification of candidates to surgical myectomy? Eur Heart J Cardiovasc Imaging. 2016; 17: 611-612.

34 Gersh BJ, Maron BJ, Bonow RO, et al. 2011 ACCF/AHA guideline for the diagnosis and treatment of hypertrophic cardiomyopathy. Circulation. 2011; 124: 2761-2796.

35 Ommen SR, Nishimura RA. Hypertrophic cardiomyopathy - one case per year? A clarion call to do what is right. JAMA Cardiol. 2016; 1: 333-334.

36 Maron BJ, Dearani JA, Maron MS, et al. Why we need more septal myectomy surgeons: an emerging recognition. J Thorac Cardiovasc Surg. 2017; 154: $1681-1685$

37 ten Cate FJ, Soliman OI, Michels M, et al. Long-term outcome of alcohol septal ablation in patients with obstructive hypertrophic cardiomyopathy: a word of caution. Circ Heart Fail. 2010; 3: 362-369. 